\title{
A Statistical Parts-Based Appearance Model of Inter-subject Variability
}

\author{
Matthew Toews ${ }^{1}$, D. Louis Collins ${ }^{2}$, and Tal Arbel ${ }^{1}$ \\ ${ }^{1}$ Centre for Intelligent Machines, McGill University, Montréal, Canada \\ (mtoews, arbel)@cim.mcgill.ca \\ ${ }^{2}$ McConnell Brain Imaging Centre, Montreal Neurological Institute, \\ McGill University, Montréal, Canada \\ louis@bic.mni.mcgill.ca
}

\begin{abstract}
In this article, we present a general statistical parts-based model for representing the appearance of an image set, applied to the problem of inter-subject MR brain image matching. In contrast with global image representations such as active appearance models, the partsbased model consists of a collection of localized image parts whose appearance, geometry and occurrence frequency are quantified statistically. The parts-based approach explicitly addresses the case where one-to-one correspondence does not exist between subjects due to anatomical differences, as parts are not expected to occur in all subjects. The model can be learned automatically, discovering structures that appear with statistical regularity in a large set of subject images, and can be robustly fit to new images, all in the presence of significant inter-subject variability. As parts are derived from generic scale-invariant features, the framework can be applied in a wide variety of image contexts, in order to study the commonality of anatomical parts or to group subjects according to the parts they share. Experimentation shows that a parts-based model can be learned from a large set of MR brain images, and used to determine parts that are common within the group of subjects. Preliminary results indicate that the model can be used to automatically identify distinctive features for inter-subject image registration despite large changes in appearance.
\end{abstract}

\section{Introduction}

Consider the task of inter-subject registration, i.e. determining correspondence between images of different subjects of a population. The task is one of great importance to the medical imaging community, as it lies at the heart of understanding how individuals vary within a population, but at the same time poses a significant challenge due to the problem of inter-subject variability. No two subjects are identical - structures may exhibit significant variation from one subject to the next or may simply not exist in all subjects. It is reasonable to expect that approaches attempting to determine a one-to-one mapping between 
different subjects may perform poorly in locations where such a mapping may not exist. Authors have attempted to identify points or regions where registration can be expected to perform well. Salient feature detection approaches are an option [89], but are generally ineffective due to inter-subject variability, as features extracted in one subject may not exist in the other. Features can be identified over a set of aligned subjects [5], but again one cannot know if these features will be relevant to new images.

We hypothesize that in order to determine the image regions likely to register well, one must first learn the degree of variability to be expected within the imaging context, and bring this knowledge to bear when registering new images. In this paper, we present a new probabilistic approach to automatically identifying image regions likely to result in meaningful inter-subject registration. Our approach is two-fold. First, off-line, we present a learning approach to building a parts-based appearance model of the image set in question (e.g. MR brain images). The model statistically quantifies the appearance, geometry and occurrence frequency of localized image parts which occur with statistical regularity over a set of training subjects. Second, we fit this model to new images to be registered, thereby identifying instances of learned model parts in the images. Statistically distinct image regions arising from the same model parts in both images are good candidates for registration.

The main contribution of this paper is a new parts-based statistical model of appearance that is based on localized image regions, as opposed to the global modes of image variation popular in the medical imaging literature [310]. The local nature of the method makes it possible to explicitly model situations where one-to-one correspondence does not exist between subjects due inter-subject variation, as model parts are not expected to (and typically do not) occur in all subjects. The model can be learned from large set of training images via a fully automatic machine learning algorithm and robustly fit to new images, all in the presence of significant inter-subject variation. The second contribution is a method of using this model to automatically determine the regions most likely to lead to meaningful registration between new images.

Our model is based on generic scale-invariant image features, and is general enough to be applied in a wide variety of image contexts. We present experiments based on T1-weighted MR brain images from the ICBM152 data set [2, which consists of 152 volumes of 88 male and 66 female normal subjects, aged $24.6 \pm 4.8$ years. Preliminary results indicate that learning is able to automatically identify a set of image parts, which can be used to determine commonality in new subjects exhibiting significant differences in appearance. The remainder of this paper is organized as follows: in Section 2 we present the parts-based model, in Section 3 we describe model learning and fitting, in Section 4 we present experimentation involving inter-subject registration, and in Section 5 we conclude with a discussion. 


\section{A Statistical Parts-Based Appearance Model}

Identifying the regions most likely to match well between two different subjects requires first learning what image structure occurs with regularity in the particular imaging context, including the range of geometrical and appearance variability to be expected. To this end, we present a statistical model based on localized image parts, which here are image regions derived from generic, automatically detected scale-invariant features. A model based on such features is attractive because it can be automatically applied in a wide variety of contexts, as opposed to approaches based on special-purpose detectors for specific image structures (i.e. a particular sulcus) or manual landmark selection, which is tedious for large data sets and prone to inter-rater variation, as a human must decide which landmarks are optimal, how many landmarks must be used, etc.

Scale-invariant features [8] offer an improvement over simple features such as corners [6], as they localize salient image patterns in scale in addition to image translation. They can be extracted in a wide variety of image contexts, and are robust to variation in intensity, in addition to geometrical deformations such as translation, orientation, scale and affine transformations. Detection and correspondence of scale-invariance features are both fast: features can be extracted efficiently from image pyramids and matched after normalizing feature image content with respect to feature geometry, removing the need to perform an explicit search over deformation parameters. Feature geometrical parameters such as location $x$, orientation $\theta$ and scale $\sigma$ recovered in the extraction process can be used formulate multiple independent hypotheses as to the geometrical transform between images, leading to occlusion and noise-resistant correspondence. In addition, scale-invariant features can be extracted from a variety of different image properties such as blobs [8], edges [9], phase [1] and entropy [7], and used in conjunction with each other.

Despite their attractive properties, scale-invariant features are rarely used for inter-subject registration because of the fact that, in general, the same features cannot be extracted in images of different subjects due to inter-subject variability. As a result, the number of features located is typically insufficient for reliably determining correspondence between different subjects. Although this fact can be considered a shortcoming of automatic feature detection, we argue in this paper that it merely reflects the difficulty of inter-subject registration, and indicates that one-to-one correspondence may not generally exist between all subjects. Recently, statistical models of features learned from a set of images have emerged in order to address these difficulties 4,12. The parts-based model presented in this section is based on statistically quantifying the occurrence frequency, appearance and geometry of features over a large set of subjects, thereby learning a set of parts that can be reliably matched between images of different subjects.

\subsection{Model Components}

Our parts-based model consists of a set of parts $m_{i}$ within a common reference frame $o$. Model parts are scale-invariant features denoted as $m_{i}:\left\{m_{i}^{b}, m_{i}^{g}, m_{i}^{a}\right\}$ 
representing the occurrence, geometry and appearance of a scale-invariant feature within an image. Feature occurrence $m_{i}^{b}$ is a binary random variable representing the probability of feature presence (or absence) in an image. Feature geometry $m_{i}^{g}:\left\{x_{i}, \theta_{i}, \sigma_{i}\right\}$ is an oriented region in $\mathbb{R}^{N}$ image space, represented by $N$-parameter location $x_{i}$, an $N-1$ parameter orientation $\theta_{i}$, and a scale $\sigma_{i}$. Feature appearance $m_{i}^{a}$ describes the image content at region $m_{i}^{g}$, and can generally be parameterized in a number of ways, such as principle components.

Reference frame $o:\left\{o^{b}, o^{g}\right\}$ represents the occurrence and geometry of a common reference frame relating parts. $o^{b}$ is a binary random variable indicating the presence/absence of the reference frame, who's significance will be made clear in the discussion of model learning and fitting. $o^{g}$ is parameterized in the same manner as scale-invariant feature geometry $\mathrm{m}^{g}$, and serves as a common geometrical frame within which different features $m_{i}$ and $m_{j}$ can be considered as statistically independent (i.e. knowing $o^{g}$, feature variation is localized to a neighborhood around an expected value of $m_{i}$ ). Within the context of MRI brain registration, a well-known definition of $o^{g}$ is the midplane line defining the Talairach stereotactic space [11, which passes from the superior aspect of the anterior commissure to the inferior aspect of the posterior commissure. Figure 1 illustrates the relationship between $o$ and $m_{i}$.
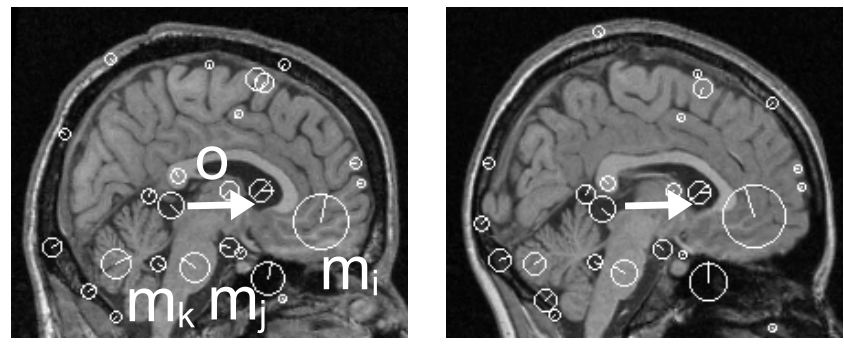

Fig. 1. Scale-invariant features and reference frames in sagittal slices of T1-weighted MR brain images of two subjects. Features $m_{i}$, illustrated as white circles inset by radial lines, are oriented regions consisting of a location $x_{i}$, orientation $\theta_{i}$ and scale $\sigma_{i}$. Reference frame $o$, illustrated as a white arrow, represents the projection of the Talairach AC-PC line onto the slice. Feature occurrence, appearance and geometry with respect to the reference frame can be quantified statistically via a parts-based model learned from a large set of subjects.

\subsection{Probabilistic Model Formulation}

Our model consists of a set of $N$ model parts $\left\{m_{i}\right\}$, which when observed in a new image can be used infer the reference frame $o$. Assuming that parts $m_{i}$ are conditionally independent given $o$, the posterior probability of $o$ given $\left\{m_{i}\right\}$ can be expressed using Bayes rule as:

$$
p\left(o \mid\left\{m_{i}\right\}\right)=\frac{p(o) p\left(\left\{m_{i}\right\} \mid o\right)}{p\left(\left\{m_{i}\right\}\right)}=\frac{p(o) \prod_{i}^{N} p\left(m_{i} \mid o\right)}{p\left(\left\{m_{i}\right\}\right)},
$$


where $p(o)$ is a prior over reference frame geometry and occurrence and $p\left(m_{i} \mid o\right)$ is the likelihood of feature $m_{i}$ given $o$. Our model focuses principally on the likelihood term $p\left(m_{i} \mid o\right)$, which can be expressed as:

$$
p\left(m_{i} \mid o\right)=p\left(m_{i}^{a}, m_{i}^{b} \mid o\right) p\left(m_{i}^{g} \mid o\right)=p\left(m_{i}^{a} \mid m_{i}^{b}\right) p\left(m_{i}^{b} \mid o^{b}\right) p\left(m_{i}^{g} \mid o^{b}, o^{g}\right),
$$

under the assumptions that $m^{a}$ and $m^{b}$ are statistically independent of $m^{g}$ given $o$, and that $m^{a}$ and $o$ are statistically independent given $m^{b}$.

Appearance likelihood $p\left(m_{i}^{a} \mid m_{i}^{b}\right)$ is represented as a multivariate Gaussian distribution in an appearance space and parameterized by mean and covariance $\mu_{i}^{a}, \Sigma_{i}^{a} \cdot p\left(m_{i}^{b} \mid o^{b}\right)$ is the probability of feature occurrence given reference frame occurrence, represented as a discrete multinomial distribution with event count parameters $\pi_{i}=\left\{\pi_{i}^{1}, \ldots, \pi_{i}^{4}\right\}$. Geometry likelihood $p\left(m_{i}^{g} \mid o^{b}, o^{g}\right)$ models the residual error of a linear transform from feature to reference frame geometry $m_{i}^{g} \rightarrow o^{g}$, and is represented as a Gaussian distribution with mean and covariance parameters $\mu_{i}^{g}, \Sigma_{i}^{g}$. In order to characterize geometrical error in a scale-invariant manner, scale is transformed logarithmically, and translation is normalized by reference frame scale.

\section{Model Learning and Fitting}

Model learning involves estimating the parameters of the set of $N$ features $\left\{m_{i}\right\}$ introduced in the previous section from a set of training images. Learning is based on a set of data vectors of the form $\left\{m_{i}^{a}, m_{i}^{g}, o^{g}\right\}$, where $m_{i}^{a}$ and $m_{i}^{g}$ are automatically extracted features and $o^{g}$ is the labeled reference frame, here taken to be the AC-PC line of the Talairach stereotactic space [11. Labeling $o^{g}$ can be done by manually defining a line segment corresponding to $o^{g}$ in images, or in an approximate manner via linear registration of $\mathrm{MR}$ volumes into the same stereotactic space, here we adopt the latter approach. Features are extracted and represented using the SIFT (scale-invariant feature transform) technique [8], based on an efficient implementation available online from the author, although a variety of other techniques could be used. Briefly, SIFT features are extracted as maxima/minima in a difference-of-Gaussian scale space pyramid, determining feature geometry $m_{i}^{g}$. The SIFT appearance representation $m_{i}^{a}$ is a 128 -value vector, corresponding to bins of a histogram of image first derivatives quantized into $8 \mathrm{x} 4 \mathrm{x} 4=128$ bins over orientation and $(\mathrm{x}, \mathrm{y})$ position.

Prior to learning, feature geometries are normalized spatially wrt the reference frame $\left\{m_{i}^{a}, m_{i}^{g}, o^{g}\right\} \rightarrow\left\{m_{i}^{a}, \bar{m}_{i}{ }^{g}\right\}$. Learning begins by clustering data vectors according to normalized geometry, thereby determining $\mu_{i}^{g}, \Sigma_{i}^{g}$. Occurrence and appearance parameters $\pi_{i}$ and $\mu_{i}^{a}, \Sigma_{i}^{a}$ are then estimated simultaneously such that the likelihood ratio $\frac{p\left(m_{i}^{b=1} \mid o^{b=1}\right)}{p\left(m_{i}^{b=1} \mid o^{b=0}\right)}$ is maximized. Note that this ratio represents a measure of the distinctiveness of a particular feature within the reference frame. As the number of clusters $N$ is unknown a priori, clustering is achieved by growing variance estimates around individual vectors, instead of through algorithms such as K-means. After learning, features can be automatically ranked according 


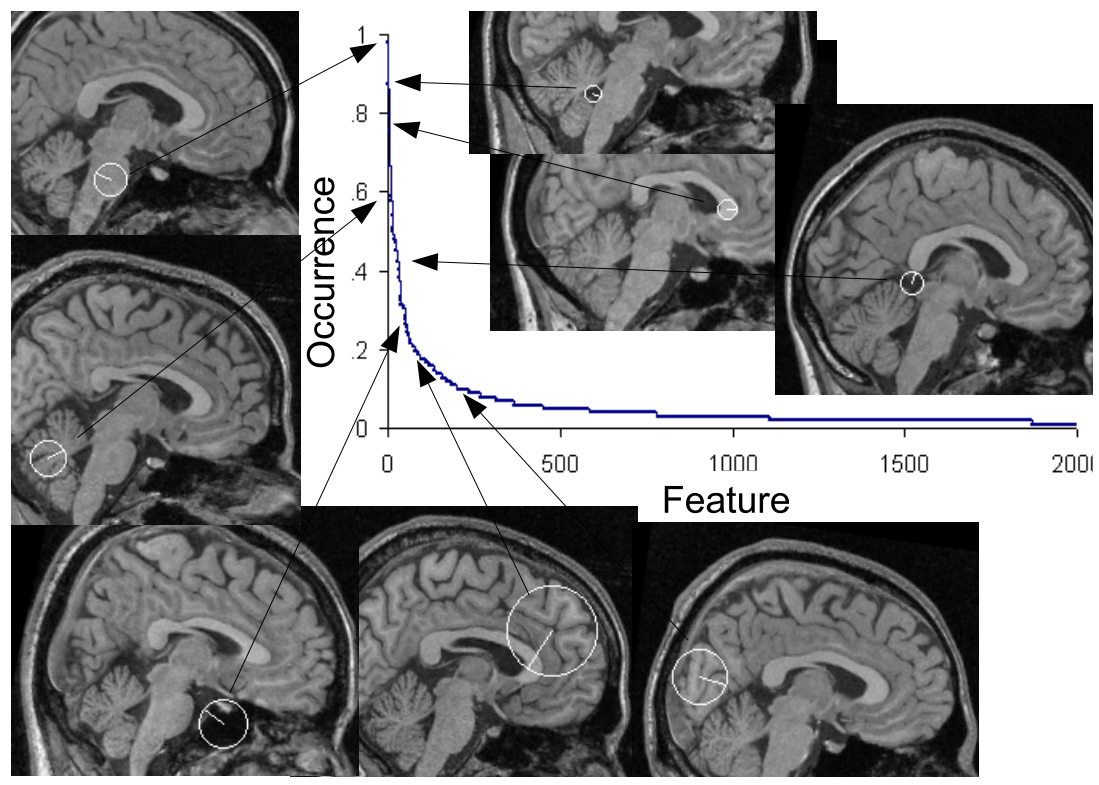

Fig. 2. A graph of features sorted by occurrence frequency $p\left(m_{i}^{b=1} \mid o^{b=1}\right)$. The images illustrate features occurring at indicated frequency within a sample of 102 sagittal slices brains from the ICBM152 data set [2]. Note that feature occurrence drops off sharply, indicating that a relatively small number of features are common to all brains, whereas a large number of features are specific to a small number of brains.

to distinctiveness or occurence frequency, as illustrated in Figure 2 Having quantified part appearance, geometry and occurrence frequency, features observed in new images can be fit to the model and assessed as to their usefulness for tasks such as inter-subject registration. Highly distinctive features indicate good candidate regions for registration, whereas poorly distinctive features represent ambiguous or subject-specific characteristics.

Once the model has been learned, it can be fit to a new image by localizing the reference frame $o$. Unlike other registration/fitting techniques based on iterative algorithms which tend to go awry when started outside a 'capture radius' of the optimal solution, our model can be fit globally. In order to determine $o$ in a new image, features extracted from the new image are matched to those in the model. Each pairing of an image feature to a model feature results in a hypothesis as to $o^{g}$ in the new image. We are interested in evaluating whether these hypotheses are the result of a true model instance or random noise, i.e. $o=\left\{o^{g}, o^{b=1}\right\}$ or $\bar{o}=\left\{o^{g}, o^{b=0}\right\}$. These two possibilities can be compared via a Bayes decision ratio:

$$
\gamma(o)=\frac{p\left(o \mid\left\{m_{i}\right\}\right)}{p\left(\bar{o} \mid\left\{m_{i}\right\}\right)}=\frac{p(o)}{p(\bar{o})} \prod_{i=1}^{N} \frac{p\left(m_{i} \mid o\right)}{p\left(m_{i} \mid \bar{o}\right)}
$$


where high $\gamma(o)$ indicates the presence of a model, and $\frac{p(o)}{p(\bar{o})}$ is a constant expected ratio of true to false model instances. Fitting is performed by identifying $o^{g}$ maximizing $\gamma(o)$, by considering clusters of model features that support similar hypotheses.

\section{Application to Inter-subject Registration}

Intuitively, fitting the model to a new image can be seen as describing the new image in terms of a unique combination of learned model parts. Images of different subjects can be compared in terms of the model parts that they share, the key notion being that any given pair of subjects shares a unique set of image parts. This information can potentially be used in a number of ways, for example to drive inter-subject registration in regions where images are known to have statistically similar content, or to cluster subject images that share similar image content. In this section, we wish explore how the features derived from the model can be applied to the task of inter-subject registration.

To apply the model to inter-subject image registration, we examine several cases where the model parts automatically learned through training are matched between new subjects not used in model learning. First, the model is fit individually to each new subject to be registered, identifying model features present in each subject. Then 10 model features of one subject are selected to be registered to the next, in two different ways: 1) selecting the most distinct model features in one subject similar to the strategy proposed by [5] and 2) selecting the most distinct model features common to both subjects. Registration is then performed by determining feature displacements from one image to the
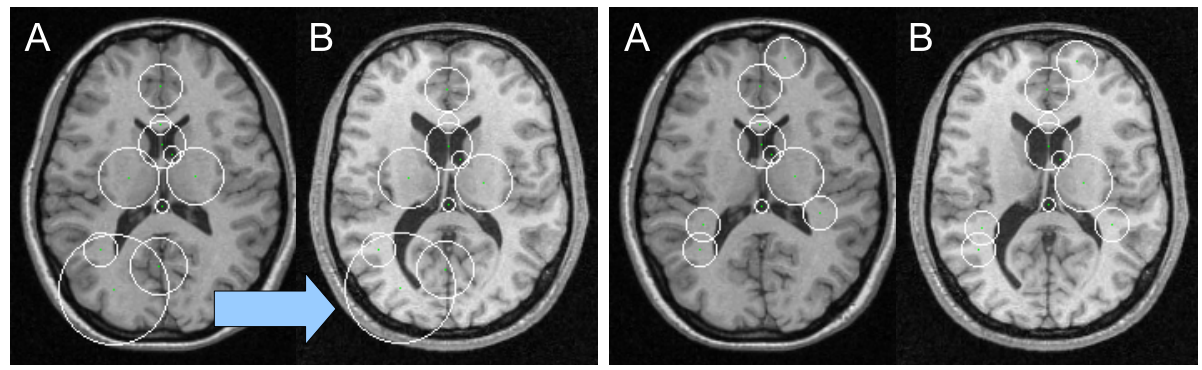

Fig. 3. Illustrating model-based feature selection for inter-subject registration. In each image pair, 10 features from subject $\mathrm{A}$ are selected in to be registered to subject B. On the left, the most distinct model features of subject A are used, on the right the most distinct features common to both subjects A and B are used. Notice that a valid registration solution may not exist in the lower left region, due to an enlarged ventricle of subject B. Basing registration on features common to both subjects, this ambiguous region can be avoided, resulting in more meaningful registration in the presence of inter-subject variability. 
next, based on a sum-of-squared-difference similarity measure regularized by an elastic prior between features. When registering normal subjects, both feature selection techniques result in reasonable registration, as most subjects share similar features. In the presence of significant inter-subject variability however, selecting features common to both subjects allows registration to avoid regions in which a valid solution may not exist, such as the enlarged ventricle illustrated in Figure 3 .

\section{Discussion}

In this paper, we presented a statistical parts-based appearance model, where a set of images is represented by a collection of automatically-extracted generic parts. The parts-based approach is unique in that it models variation on a local scale, and as such is able to explicitly model situations where one-to-one correspondence does not exist between subjects. Experimentation showed that model parts can be identified and quantified statistically by a fully automatic learning algorithm, and robustly fit to new images, all in the presence of significant intersubject variability. In addition, preliminary results demonstrated that the model can be used to identify statistically distinct parts common to different subjects, indicating image structure that can be matched with higher reliability for the task of inter-subject registration. In general, parts-based modeling represents a powerful tool for understanding the variation in local anatomical structure over a large set subjects, and offers many avenues for future exploration. Part statistics could potentially serve as indicators of abnormal conditions such as pathology. A parts-based framework could potentially be used to relate anatomical structures across different species. These encouraging results and future directions should naturally lead to the development of robust scale-invariant feature detectors in $3-\mathrm{D}$ and $4-\mathrm{D}$, and the application of the model in a wide variety of contexts.

\section{References}

1. G. Carneiro and A.D. Jepson, Multi-scale phase-based local features, CVPR, vol. 1, 2003, pp. 736-743.

2. D. L. Collins, N.J Kabani, and A.C. Evans, Automatic volume estimation of gross cerebral structures, 4th International Conference of Functional Mapping of the Human Brain (A. Evans, ed.), 1998.

3. T.F. Cootes, G.J. Edwards, and C.J. Taylor, Active appearance models, Pattern Analysis and Machine Intelligence 23 (2001), no. 6, 681-684.

4. R. Fergus, P. Perona, and A. Zisserman, Object class recognition by unsupervised scale-invariant learning, CVPR, 2003, pp. 264-271.

5. Wu Guorong, Feihu Qi, and Dinggang Shen, Learning best features for deformable registration of $m r$ brains, MICCAI, 2005.

6. C. Harris and M. Stephens, A combined corner and edge detector, Proceedings of the 4th Alvey Vision Conference, 1988, pp. 147-151.

7. T. Kadir and M. Brady, Saliency, scale and image description, IJCV 45 (2001), no. $2,83-105$. 
8. David G. Lowe, Distinctive image features from scale-invariant keypoints, IJCV 60 (2004), no. 2, 91-110.

9. K. Mikolajczyk and C. Schmid, Scale and affine invariant interest point detectors, IJCV 60 (2004), no. 1, 63-86.

10. Daniel Rueckert, Alejandro F. Frangi, and Julia A. Schnabel, Automatic construction of 3-d statistical deformation models of the brain using nonrigid registration, TMI 22 (2003), no. 8, 1014-1025.

11. J. Talairach and P. Tournoux, Co-planar stereotactic atlas of the human brain: 3dimensional proportional system: an approach to cerebral imaging, Georg Thieme Verlag, Stuttgart, 1988.

12. M. Toews and T. Arbel, Detection over viewpoint via the object class invariant, ICPR, 2006. 